\title{
A Novel Crossing Current Resonant Converter (XCRC)
}

\author{
N.K.Poon* M.H.Pong** \\ * BSC Electronic Division, Hong Kong \\ ** Electrical \& Electronic Engineering Department, \\ Hong Kong University, Pokfulam Road, Hong Kong
}

\begin{abstract}
Unlike other resonate or soft-switching converter, this novel topology employed only two switches and very simply control method.Inherent constant power protect feature is another merit. No special resonate chip is needed to control the circuit. By adding two diode parallel with the dividing capacitors and reconsiderate the capacitors to a small value that will fully charge and discharge in one cycle. One can obtain zero voltage-switching characteristic and control the output voltage regulation by frequency modulation. An industrial standard PWM chip can be easily modified to a VCO as the control element.
\end{abstract}

\section{INTRODUCTION}

There is a general consent that sinusoidal resonant topology are hard to control and manipulated since the resonant cycle are greatly depended on load variation. In order to build a power supply that can perform current or voltage resonance throughout the full range of load or input voltage, the turn on and turn off period should be varied according to the specified range. In general a very complex circuit should be build in order to detect the zero crossing transient. Moreover current or voltage stresses on the switching devices will be larger than the traditional PWM converter. A lot of penalty are paid for sinusoidal resonant topology.

More works are now concentrating on soft-switching topology, since it has a simpler control method but can still suppress the switching loss as well as EMI problem. One of the most successful method is the phase modulation topology. However Phase modulation configuration employs four switching elements to complete the zero voltage switching , moreover special chip set is used for controlling the circuit. As a result, engineers hesitate to use this topology in low power converter when cost become a critical consideration. The new Crossing Current Resonant Converter (XCRC) topology just fill the gap of lower power and lower cost zero voltage soft-switching technique.

\section{CIRCUIT OPERATION}

Fig. 1 shows the basic structure of the converter. The schematic of the circuit shows the difference between this topology and a half bridge converter is the two diodes across the dividing capacitors. Despite the similarities the operation principle is totally different from the tradition half bridge topology. The two voltage dividing capacitors are so small that it will be charged up to the rail voltage or discharge to $0 \mathrm{~V}$ while one switch is on.

By considering the primary inductor $\mathrm{Lr}$ current loop, the circuit has three working states. Namely 1) Power transfer state, 2) Current circulating state, 3) Crossover resonant state.

By symmetry one can consider a half cycle operation to explain the above states. 


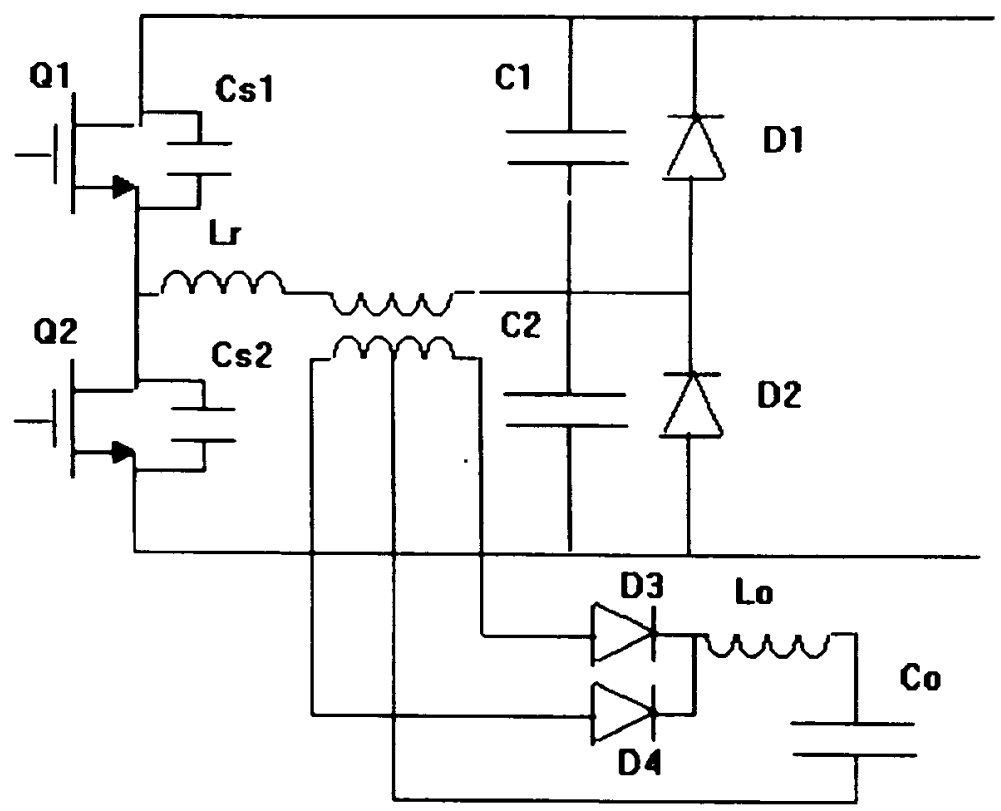

Fig. 1

A) Power transfer state

In Fig. 2, while $\mathrm{Ql}$ is on, current will flow through the transformer and charge up $C 2$ until it reaches the supply voltage. During this state, the transformer will "see" a voltage applied on it's terminals, so secondary voltage will be built and power will be transfer from the primary to the secondary and filtered by the output filter. The voltage across the capacitor $\mathrm{C2}$ canno be charged up anymore because the diode D1 will clamp the $\mathrm{Cl}$ voltage to the supply voltage and force the circuit into "Freezing State".

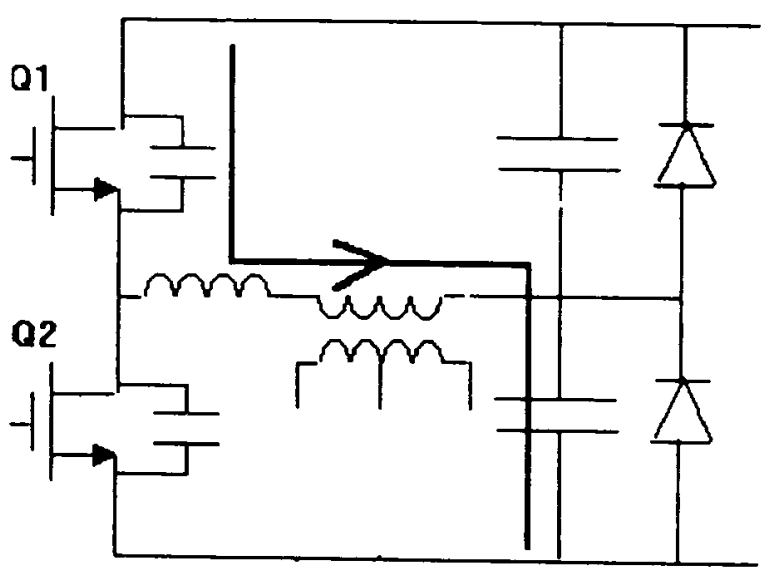

Fig. 2
B) Current circulating state

In Fig. 3 ,While Q1 is still on, the primary current can still form a loop by flowing through the diode, and the voltage across the transformer and the series inductor $\mathrm{Lr}$ will dropped to approximately zero voltage. Regarding the circuit it is assumed that voltage is zero voltage in practice. As a basic physic law the $\frac{d i}{d t}$ of the inductor will be equal to zero, as a result the current will be frozen during that state until Q1 is off.

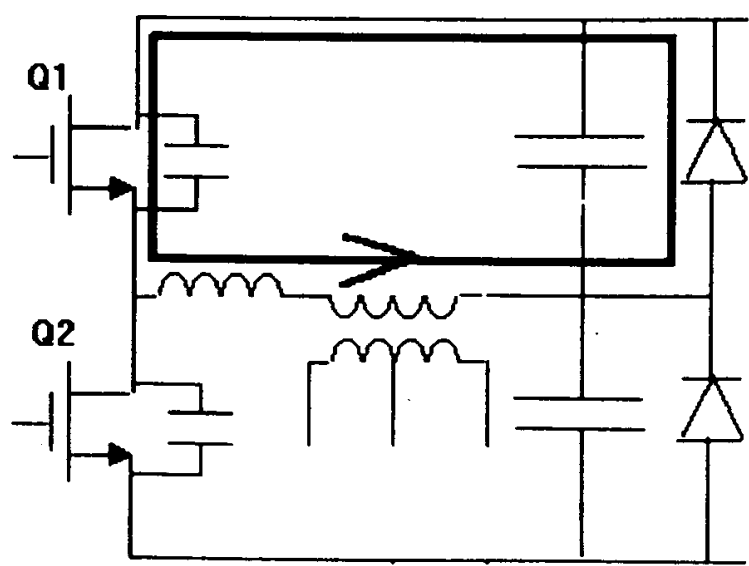

Fig. 3 
C) Crossover resonant state

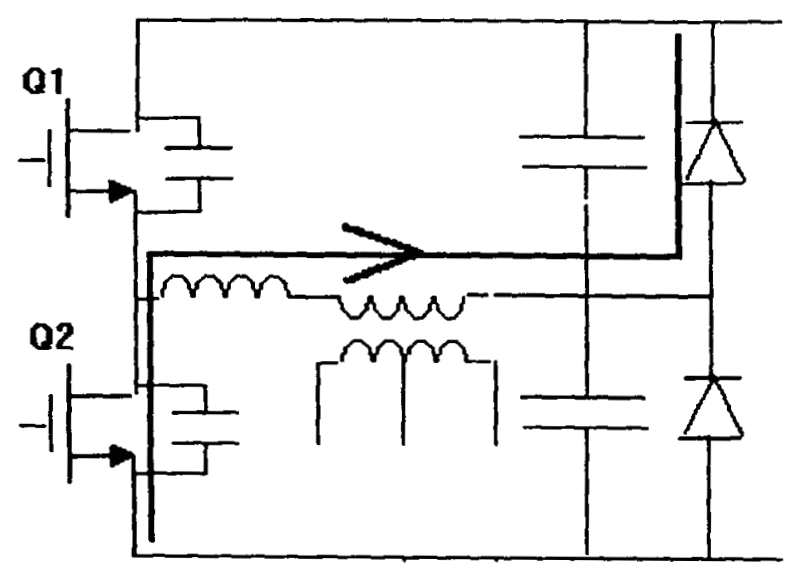

Fig. 4

In Fig. 4, While $Q 1$ and $Q 2$ is off, a dead time is now introduced, the primary current will discharge the Cs2 of the Q2 to $-0.7 \mathrm{~V}$ and current will then flow through the body diode of the MOSFET Q2. While the body diode of Q2 is forward conducting, Q2 will turn on which starts the next half period of the cycle. And it results in a zero voltage tum on of both switches and the process repeat symmetrically.

\section{D) Woveform illustration}

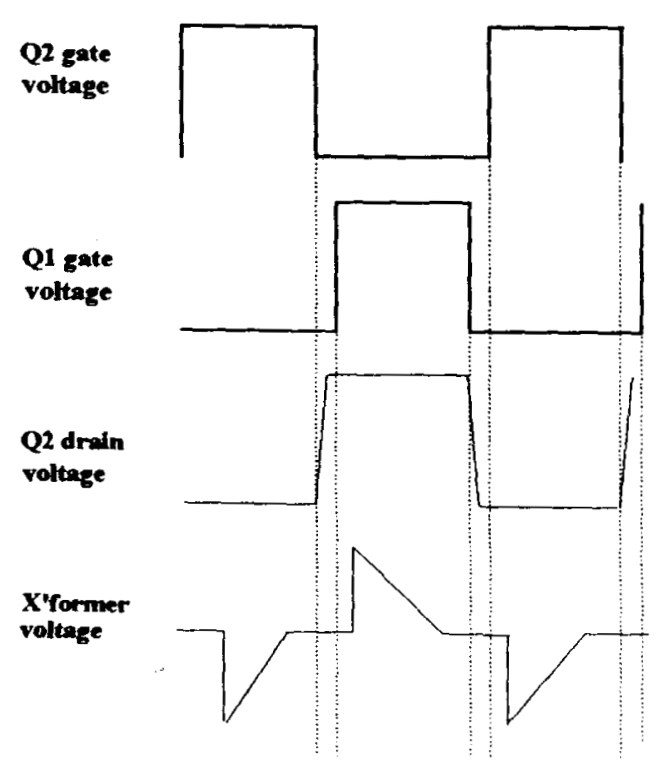

Fig. 5
The gate driving waveform are two alternating wave with a constant dead time squarewave, while the dead time is compared small with the switching period, the output voltage is a ratio of average value of the transformer primary voltage. The voltage of the transformer will gradually drop to zero voltage because the dividing capacitor will charge up to the supply voltage and cause no potential different between the transformer and the series inductor. Although the gate drive pulse is still pushing high no voltage will be applied to the transformer's primary. By adjusting the frequency, one can adjust the average voltage of the transformer as well as the output voltage.

\section{DETERMINATION OF COMPONENT VALUES}

\section{A) Maximum output power:}

It is quite obvious to see that the output power depended on how much energy store in the dividing capacitors that discharge completely at each cycle. The relationship can be shown below

$$
\text { Maximum Output power }=2 \mathrm{CV} V_{\text {in }}^{2} f_{\max }
$$

\section{B) Minimum series inductor $\mathrm{Lr}$}

To ensure zero voltage switching, there, must be enough energy to charge up the MOSFET stray capacitors Cs reach to the supply rail voltage, the minimum inductance can be expressed as

$$
\text { Minimum Inductance } \mathrm{Lr}=\frac{2 C_{s} V_{\text {in }}^{2}}{I_{\bar{p}}^{2}} \text {, }
$$

Where Ip is primary transformer current that is determined by the output power.

\section{C) Allowable dead time}

During the dead time, the circuit is in the crossover resonant state, and in fact the dead time consists of three separated periods that are determined by the series resonant inductor $\mathrm{Lr}$, 


$$
\text { . NT }=\frac{i}{\sqrt{2 L_{r} C}} \text {, }
$$

In Fig. 6 ,

$$
\mathrm{Ta}=\sqrt{2 C_{s} L_{r}} \sin ^{-1}\left(\frac{V_{\text {in }} \sqrt{2 C_{s}}}{I_{p} \sqrt{L_{r}}}\right)
$$

where $\mathrm{Ta}$ is the time for the primary current to swing to the supply source, and once the voltage it introduces on the stray capacitance of the MOSFET reaches the supply voltage the second period of the dead time is controlled by $\mathrm{Tb}$ as follow,

$$
\mathrm{T}_{\mathrm{b}}=\frac{L_{r} I_{p} \cos \left(\omega T_{a}\right)}{V_{\text {in }}},
$$

The ending of $\mathrm{Tb}$ is caused by the exhaust of the primary current or when it return to zero, and it will increase in a reverse direction as the opposite switch has already turn on,

$$
\mathrm{Tc}=\frac{1}{\omega} \sin ^{-1}\left[\frac{I_{p} L_{r} \omega}{V_{i n}}\right]
$$
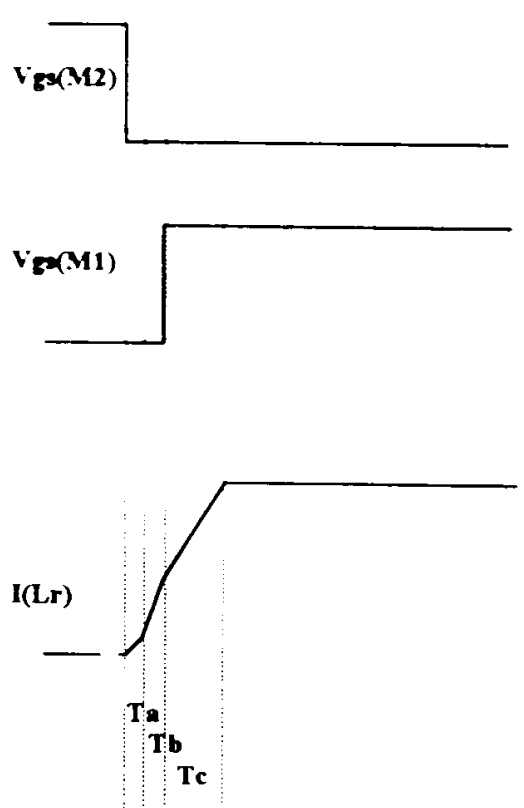

Fig. 6

\section{D) Output voltage}

In actual circuit model, the voltage is very complex and is unreasonable to express it in a exact way, however by assuming the magnetizing inductance of the transformer is large and the circuit works in continues mode, the result can be easily obtained as,

$$
V_{o}=\frac{2 C V_{s}^{2} f_{s}}{l_{o}}
$$

where

$$
V_{s}=\frac{N_{s} V_{\text {in }}}{N_{p}},
$$

\section{EXPERIMENTAL RESULT}

The experimental result matches with the theory prediction, and the merit of zero voltage switch can be demonstrated by the fact that the temperature rise of the non-heatsinked TO-220 packaged MOSFET is merely 30 degree with $40 \mathrm{~W}$ output. Fig. 7, Fig. 8 show the waveform of the circuit with a $41 \mathrm{uH}$ series inductor $\mathrm{Lr}$, and $\mathrm{C} 3, \mathrm{C} 4$ were $5.8 \mathrm{nF}$, the transformer ratio is $1: 7$,

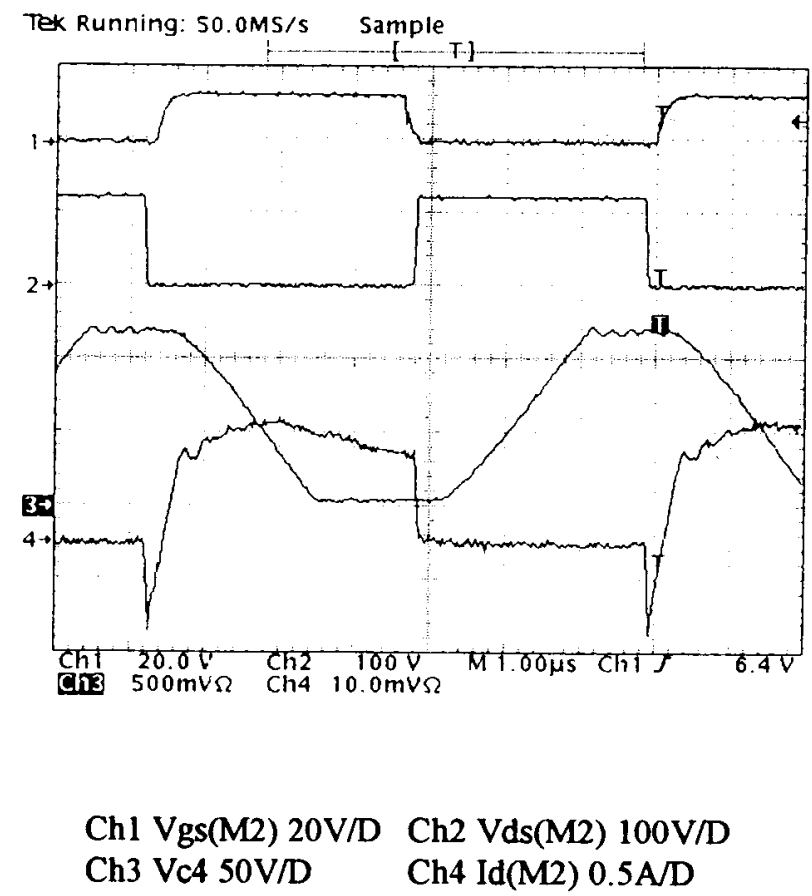

Fig. 7 Captured waveform 


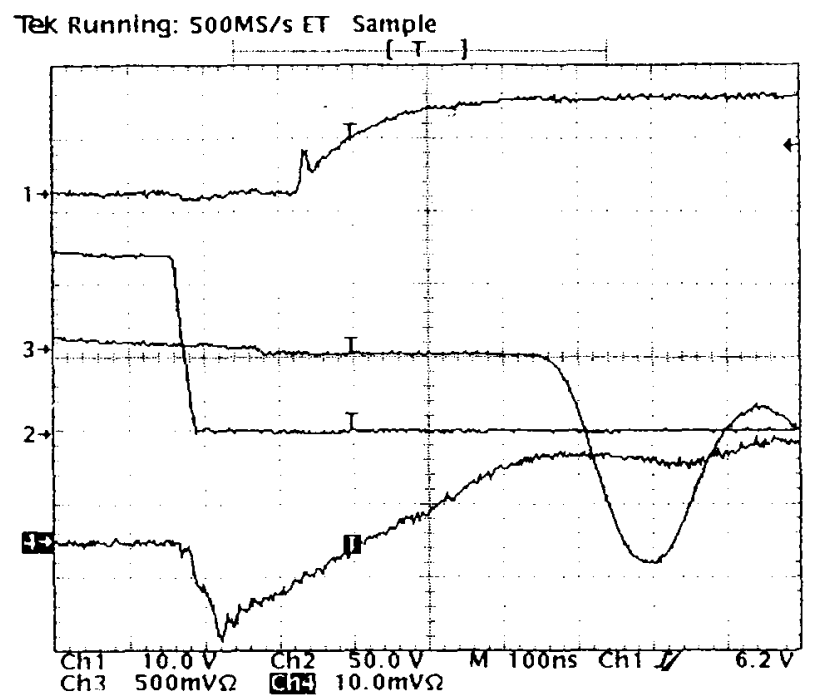

$\begin{array}{ll}\text { Ch1 Vgs(M2) 10V/D } & \text { Ch2 Vds(M2) 50V/D } \\ \text { Ch3 Vp 50V/D } & \text { Ch4 Id(M2) 0.5A/D }\end{array}$

Fig. 8 Waveform at the crossing current state

V. CONCLUSION.

A simple configuration and easy control topology has been developed. The said configuration is especially suitable for low power applications. Very low voltage and current stress present at the switching elements. The zero voltage switching characteristic result in a very high efficiency converter. The control chip can be easily modified from a industrial standard PWM IC. The design is free from any voltage or current overshoot, moreover the voltage dividing capacitors can limit the output power to provide inherent power limiting.

\section{REFERENCE}

1.)Richard Redl, Nathan O. Sokal, Laszlo Balogh, "A novel soft-switching full bridge DC/DC converter: analysis, design consideration and experimental result at $1.5 \mathrm{KW}, 100 \mathrm{KHz}$ "IEEE PESC.

2) M.H.Pong Computer aided Analysis of a Buck Zero Current Switching Resonant converter", Proceedings of the International Power Engineering conference IPEC pp.543-548, Singapore, March 1993

3) W.C.Ho, M.H.Pong "Power Loss and Efficiency Analysis of Quasi-Resonant converters" International Conference on Industrial Electronics, Control and Instrumentation IECON 93, Hawaii, November 1993. 